\title{
Research on The Step Form Selective Harmonic Elimination Technique of Cascaded H Bridge Converter
}

\author{
Ling Wang, Zhenyuan Qin, Bin Zhang \\ School of Harbin University of Science And technology,Harbin 150000,China.
}

\begin{abstract}
Keywords: non-linear harmonic elimination equation, initial value setting, modulation ratio
\end{abstract} amplitude range, power balance control

Abstract. In this paper, a simple and accurate empirical formula is adopted to establish an improved initial value setting model, and a relatively complete initial value setting method is obtained.We use a test search model with equal step size to determine the modulation ratio amplitude range $m$ that has a solution to the nonlinear harmonic elimination equation in a certain number of units.Based on the step size search model, we analyze the inclusion relation of Switching angles and the improvement effect of output voltage waveform by the increasing of the number of $n$ units when the $m$ is specified.We obtain that the modulation ratio amplitude range $m$ has a more reliable solution. The Total Harmonics Distortion is decreased with the increasing of $n$, which represents the harmonic removing effects is good. And a feasible scheme can be given in the unit power balance optimal switching control strategy. The model is general and can be extended to harmonic elimination technique in practical engineering.

\section{Introduction}

In the power system, to achieve the high voltage and multi-level output in the study, it is a more widely used method , SPWM (PWM Sinusoidal) method, to solve the Pulse Width Modulation (PWM).Using the selected harmonic elimination pulse width modulation technology( SHEPWM) of three-level cascaded H-bridge converter is a scheme to achieve the SPWM. The key to eliminate selected harmonics and to improve the waveform quality of the output voltage is to meet the desired output fundamental wave voltage vac and to select the specific switching time. The cascade multilevel converter is connected in series with some converter modules, and its basic system structure is shown in Figure 1.It is often adopted a $\mathrm{H}$ bridge converter unit with 3-level output in the converter modules, where the inductance acts as filter.

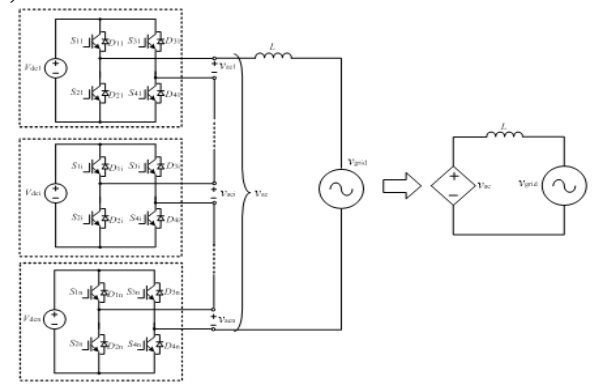

Fig.1 Grid-connected system and equivalent circuit based on cascade $\mathrm{H}$ bridge converter

By Fourier series expansion of step voltage,formulation (1) ,Fourier series of the amplitude of the s-th of the voltage $\mathrm{v}_{\mathrm{ac}}$, is derived as follow:

$$
V_{\mathrm{s}}=\frac{4 V_{d c}}{s \pi} \sum_{s=1,3,5}^{\infty}\left[\cos \left(s \theta_{1}\right)+\cos \left(s \theta_{2}\right)+\cdots \cos \left(s \theta_{n}\right)\right]
$$

where, $0 \leq \theta_{1} \leq \theta_{2} \leq \cdots \theta_{\mathrm{n}} \leq \pi / 2$.

Therefore, the methods of harmonic elimination technique is to find out a set of switching angles $\theta_{\mathrm{i}}(\mathrm{i}, 2, \cdots \mathrm{n})$, and amplitude of the output voltage fundamental component is $V_{1 m}$ and the output voltage does not contain low harmonics. The detailed methods as follows:

The amplitude modulation ratio is defined as below: 


$$
m=V_{1 m} /\left(n V_{d c}\right)
$$

Then according to the requirements of the harmonic elimination above, making the low frequency expansion equation is not exist, we derive the nonlinear algebraic equations (2) of switching angles $\theta_{\mathrm{i}}(\mathrm{i}, 2, \cdots \mathrm{n})$.

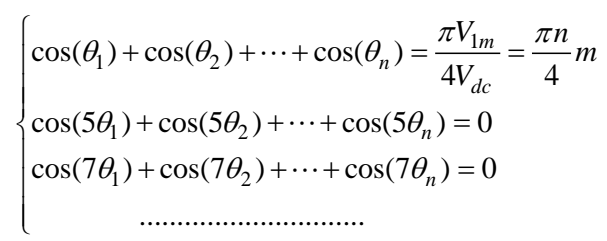

For a certain number of cascade modules $n$, nonlinear equations have a solution when modulation ratio $m$ is in a certain range of values. And it requires a set of initial values $\theta_{\mathrm{i}}^{0} \quad(\mathrm{i}=1, \cdots$, $n$ ) to meet the solution convergence of the equation group.In order to check the output voltage $V_{\text {ac }}$ waveform quality of the step form harmonic elimination technique, it can be described by the Total Harmonics Distortion. As shown in formulation (3) below.

$$
T H D=\sqrt{\frac{\sum_{i=6 k+1}^{\infty} V_{i}^{2}}{k=1,3, \ldots}} \frac{V_{1}^{2}}{k} \times 100 \%=\sqrt{\frac{V_{5}^{2}+V_{7}^{2}+V_{11}^{2}+\cdots V_{43}^{2}+\cdots}{V_{1}^{2}}} \times 100 \%
$$

Therefore,we know that if the low order harmonic output is less, the value of THD is smaller, and the better of the waveform quality is obtained.

\section{Initial value setting model}

Equations (2), no-nlinear transcendental equations, are solved using the fsolve function in MATLAB software by commonly iterative method.But, the disadvantage of this method is that it has a certain requirement for the initial value selection,because it has a direct impact on the convergence and precision of solution ${ }^{[1]}$. When the cascade number $\mathrm{n}$ is determined, it shows that $(n-1) /(n * m)>1$, thus this formulation, $\sin ^{-1}(k / m \cdot n)$, has no real solution. In order to extent the applicability of this model and still to get a reliable initial value, an empirical formula is selected to improve the model through consulting the relevant literature ${ }^{[2]}$.Note that it doesn't allow any two switching angles equal in the process of the iterative initial values,otherwise,it will occur singularity in finding the solution of linear simultaneous equations.For example, when $n$ equals 3 and $m$ is 0.5 , $m$ is beyond the range ( $m>2 / 3)$, and the results of switching angle initial values are as follows:

$$
\theta_{1}^{0}=0.5236 \quad \theta_{2}{ }^{0}=0.7854 \quad \theta_{3}{ }^{0}=1.0472
$$

Finally, we obtain the results of the switching angles as below:

$$
\theta_{1}=0.7116 \quad \theta_{2}=1.1489 \quad \theta_{3}=1.5595
$$

\section{A solution decision model based on the equal step size test}

Only given the number of the cascade units $n, m$ is variable at this time, we first consider the maximum possible range of $m$.

$$
\left\{\begin{array}{l}
V_{1 m}=\frac{4 V_{d c}}{\pi}\left[\cos \left(\theta_{1}\right)+\cos \left(\theta_{2}\right)+\ldots+\cos \left(\theta_{3}\right)\right] \\
\cos \left(\theta_{1}\right)+\cos \left(\theta_{2}\right)+\ldots+\cos \left(\theta_{n}\right)=\frac{\pi V_{1 m}}{4 V_{d c}}=\frac{\pi n}{4} m \\
m=V_{1 m} /\left(n V_{d c}\right) \\
\cos \left(\theta_{n}\right)<\cos \left(\theta_{n-1}\right)<\ldots<\cos \left(\theta_{1}\right) \leq 1
\end{array}\right.
$$

Form eqs.(4),we know $m<4 / \pi$ and $m>0$ from definition,we derive $0<m<4 / \pi$.Because each term in the equations (2) is arbitrary differentiability, it shows that the solution range of this equations is continuous. For the modulation ratio amplitude $m$ of $0 \sim 4 / \pi$,we use a idea selecting equal steps by 
increasing the value of $m$ in turn to search. Finally, we find out a value of $m$ based on the solution process. If the step size is specified as $\delta$,we have:

$$
m_{i}=m_{i-1}+\delta(\mathrm{i}=0,1,2 \ldots \mathrm{n})
$$

where $, m_{0}=0, m_{n}=(4 / \pi-\delta)$;Well, let's plug the $n$ and $m_{i}$ into the equations(2), and we determine whether $m_{i}$ has a solution. In the end, the $m_{i}$ corresponding to $\theta_{\mathrm{i}}$ by using the image, we can be obtained the solution of the $m$ range.The results show that with the increase of $n$, the lower bound of $\mathrm{m}$ is improved. But, in general, the value of $m$ decreases with the length of the interval.When $n$ is equal to15, the relations between $m$ and switch angle is as shown in figure 2 .

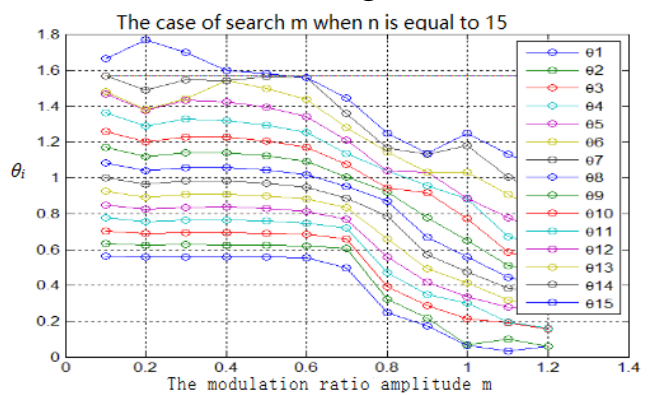

Fig. 2 The relations between $m$ and switch angle

\section{Switching angle range analysis model based on step size test}

Under the condition of having the same modulation ratio amplitude m,we consider mutually inclusive relationship between the modules and switching angles in order to facilitate the analysis.Based on the research the test search model with equal step,we obtain the clearance range $(0.64 \leq m \leq 1.05)$ of the modulation ratio amplitude $\mathrm{m}$. Then the corresponding switching angles are solved using the fslove function. Finally, we find out the mutually inclusive relationship among three set of switching angles through analysis. Considering a precedent, when the $\mathrm{m}$ is 0.85 , the corresponding switching angle is shown in figure 3 .

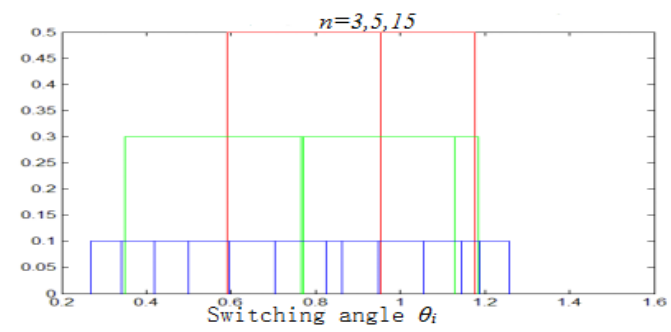

Fig.3 The mutually inclusive relationship

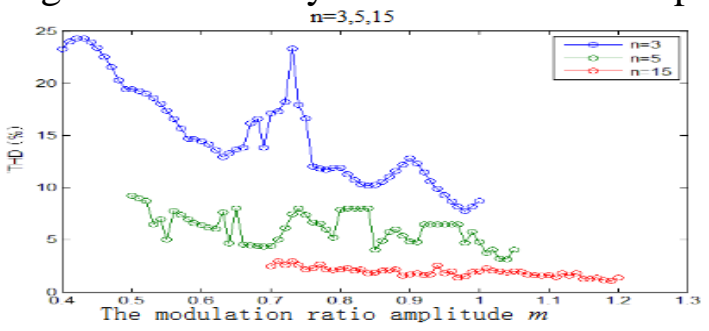

Fig.4 The graph of relationship between THD and $m$ of switching angles

Similarly, the graph of relationship between Total Harmonics Distortion THD and the modulation ratio amplitude $m$ is shown in figure 4 .

From the image above,we know that the value of Cascade number $n$ decreases with the increasing of THD. For the same value of $n$, value of THD changes slightly with the change of $m$, and the larger is the $n$,the slower is this change. With the increasing of $n$, the output voltage $V_{a c}$ waveform quality become better, more closer to the sine curve. 


\section{Power balance control model}

The fundamental amplitude $V_{c i}$ of the i-th module is below:

$$
V_{c i}=\frac{4 V_{d c}}{\pi}\left(\cos \theta_{i 1}-\cos \theta_{i 2}+\cos \theta_{i 3}\right)
$$

Module C1 output power ${ }^{[3]}$ is shown as follow:

$$
\begin{aligned}
P_{c i} & =V_{c i} i_{a n} \\
& =\frac{4 V_{d c}}{\pi}\left(\cos \theta_{i 1}-\cos \theta_{i 2}+\cos \theta_{i 3}\right) * \sin (\omega t) * I_{m} \sin (\omega t-\phi) \\
& =\frac{2}{\pi} V_{d c} I_{m}\left(\cos \theta_{i 1}-\cos \theta_{i 2}+\cos \theta_{i 3}\right)[\cos \phi-\cos (2 \omega t-\phi)]
\end{aligned}
$$

where, $i_{a n}=I_{m} \sin (\omega t-\phi)$; the symbol $\phi$ denotes power factor angle and it isn't considered by the effect of the changes. The active power $P_{c i}$ is as below:

$$
P_{c i}=\frac{2}{\pi} V_{d c} I_{m}\left(\cos \theta_{i 1}-\cos \theta_{i 2}+\cos \theta_{i 3}\right) \cos \phi
$$

From the above formulation, it shows that the magnitude of the output power $P_{c i}$ of the $i$-th unit is proportional to the fundamental wave amplitude. In order to realize the switching load balancing of each unit output power and power device, a new power balance control strategy based on SHEPWM is proposed.The strategy of using SHEPWM control angle is flexible to adjust, in order to ensure the output voltage waveform is quarter period symmetric. According to the value of the modulation, the transform unit control angle $\theta_{i j}$ is performed by flexible manipulations within quarter period, and it means that $P_{c 1=} P_{c 2}=\ldots=P_{c n}$. The new scheme controlling principle is shown in figure 5.

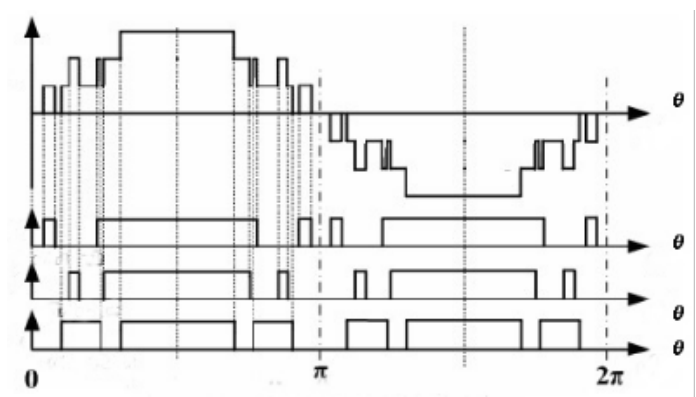

Fig.5 The new scheme controlling principle chart

For the case of $n=20$, when $m$ is 0.8 ,all units are positive before negative, then positive, in a quarter-period. Solutions of switching angle are as follow in this table. 


\section{Summary}

Table 1.Solutions of switching angle

\begin{tabular}{|c|c|c|c|}
\hline n switching angles & $\theta_{1}$ & $\theta_{2}$ & $\theta_{3}$ \\
\hline 1 & 0.07 & 0.10 & 0.10 \\
\hline 2 & 0.10 & 0.13 & 0.14 \\
\hline 3 & 0.15 & 0.19 & 0.21 \\
\hline 4 & 0.19 & 0.25 & 0.27 \\
\hline 5 & 0.25 & 0.30 & 0.33 \\
\hline 6 & 0.28 & 0.36 & 0.37 \\
\hline 7 & 0.35 & 0.50 & 0.52 \\
\hline 8 & 0.35 & 0.35 & 0.36 \\
\hline 9 & 0.42 & 0.48 & 0.49 \\
\hline 10 & 0.49 & 0.59 & 0.59 \\
\hline 11 & 0.54 & 0.56 & 0.56 \\
\hline 12 & 0.59 & 0.68 & 0.68 \\
\hline 13 & 0.63 & 0.65 & 0.66 \\
\hline 14 & 0.56 & 0.58 & 0.69 \\
\hline 15 & 0.65 & 0.67 & 0.75 \\
\hline 16 & 0.58 & 0.59 & 0.81 \\
\hline 17 & 0.77 & 0.79 & 0.87 \\
\hline 18 & 0.86 & 0.87 & 0.95 \\
\hline 19 & 0.93 & 0.95 & 1.03 \\
\hline 20 & 0.99 & 1.03 & 1.07 \\
\hline
\end{tabular}

\section{References}

[1] Shakhno S M. On a Kurchatov's method of linear interpolation for solving nonlinear equations[J]. Pamm, 2004, 4(4):650-651.

[2] Manyuan Ye, Pinggang Song, Chaowei Zhang. Study of harmonic elimination technology for multi-level inverters[C]// Industrial Electronics and Applications, 2008. Iciea 2008. IEEE Conference on. IEEE, 2008:2738-2741.

[3] Jinping chen, On Novel Topology and Modulation Strategy for Multilevel DC-link Inverter [J].Chang'an University.

[4] Yi Su, The Solutions of the Switching Angles for Half-Wave Symmetry SHEPWM[C].WuHan University,2012.

[5] Sun Y F, Xin-Bo R. Power Balance Control Schemes for Cascaded Multilevel Inverters[J]. Proceedings of the Csee, 2006, 26(4):126-133. 